\title{
Sandwiching the Consistency Strength of Two Global Choiceless Cardinal Patterns \\ by
}

\author{
Arthur W. APTER \\ Presented by Czestaw BESSAGA
}

\begin{abstract}
Summary. We provide upper and lower bounds in consistency strength for the theories "ZF $+\neg \mathrm{AC}_{\omega}+$ All successor cardinals except successors of uncountable limit cardinals are regular + Every uncountable limit cardinal is singular + The successor of every uncountable limit cardinal is singular of cofinality $\omega$ " and " $\mathrm{ZF}+\neg \mathrm{AC}_{\omega}+$ All successor cardinals except successors of uncountable limit cardinals are regular + Every uncountable limit cardinal is singular + The successor of every uncountable limit cardinal is singular of cofinality $\omega_{1}$ ". In particular, our models for both of these theories satisfy "ZF $+\neg \mathrm{AC}_{\omega}+\kappa$ is singular iff $\kappa$ is either an uncountable limit cardinal or the successor of an uncountable limit cardinal".
\end{abstract}

There are many instances in the literature where choiceless large cardinal patterns are initially forced from strong hypotheses which one later sees can be weakened somewhat. For example, it is shown in [4] that the models constructed in [11] from an almost huge cardinal can actually be built from a cardinal which is intermediate in consistency strength between a supercompact limit of supercompact cardinals and an almost huge cardinal.

The purpose of this paper is to continue in this vein by proving two "sandwich theorems", where a sandwich theorem traps the consistency strength of a particular statement between two distinct large cardinal axioms. In particular, we begin by providing a smaller new upper bound in consistency strength and a new lower bound in consistency strength for a choiceless cardinal pattern that is a corollary of the work of [11] and [4]. We then show how the methods developed can be used to prove a sandwich theorem for a

2010 Mathematics Subject Classification: 03E25, 03E35, 03E45, 03E55.

Key words and phrases: supercompact cardinal, supercompact Radin forcing, Radin sequence of measures, symmetric inner model. 
choiceless cardinal pattern that does not follow explicitly from the work of 11] and [4]. Specifically, we have the following theorems.

THEOREM 1.

(1) Suppose $V \vDash " Z F C+\kappa$ is $2^{\left[\beth_{\omega}(\kappa)\right]^{<\kappa}}$ supercompact". There is then a partial ordering $\mathbb{P} \in V$ and a symmetric submodel $N \subseteq V^{\mathbb{P}}$ of height $\kappa$ such that $N \vDash " Z F+\neg A C_{\omega}+$ All successor cardinals except successors of uncountable limit cardinals are regular + Every uncountable limit cardinal is singular + The successor of every uncountable limit cardinal is singular of cofinality $\omega "$.

(2) Assume $Z F$ and that all uncountable limit cardinals are singular and the successor of every uncountable limit cardinal is singular of cofinality $\omega$. Then for every $n<\omega$ and every set of ordinals $x, M_{n}^{\sharp}(x)$ exists.

\section{THEOREM 2.}

(1) Suppose $V \vDash " Z F C+\kappa$ is $2^{\left[\beth_{\omega_{1}}(\kappa)\right]^{<\kappa}}$ supercompact". There is then a partial ordering $\mathbb{P} \in V$ and a symmetric submodel $N \subseteq V^{\mathbb{P}}$ of height $\kappa$ such that $N \vDash " Z F+\neg A C_{\omega}+$ All successor cardinals except successors of uncountable limit cardinals are regular + Every uncountable limit cardinal is singular + The successor of every uncountable limit cardinal is singular of cofinality $\omega_{1}$ ".

(2) Assume $Z F$ and that all uncountable limit cardinals are singular and the successor of every uncountable limit cardinal is singular of cofinality $\omega_{1}$. Then for every $n<\omega$ and every set of ordinals $x, M_{n}^{\sharp}(x)$ exists.

We note that Theorems $1(2)$ and 2(2) have an additional strong consequence. This is that for every $n<\omega$, there is an inner model with $n$ Woodin cardinals, i.e., that PD holds in all set generic extensions. Theorems 1(2) and 2(2) are due to Busche and Schindler [7]. They follow from [7, Section 3.1], and are corollaries of [6, Theorem 5].

We will explicitly prove Theorem 1. However, since the proof of Theorem 2 is virtually identical to the proof of Theorem 11, we will only indicate the minor modifications to the proof of Theorem 1 that need to be made in order to establish Theorem 2. Also, we observe that our models witnessing the conclusions of Theorems 1(1) and 2(1) satisfy the theory " $\mathrm{ZF}+\neg \mathrm{AC}_{\omega}$ $+\kappa$ is singular iff $\kappa$ is either an uncountable limit cardinal or the successor of an uncountable limit cardinal".

Our proof uses Gitik's techniques of [11]. Our presentation of Gitik's techniques is based on the one given in [3], but also follows the ones given in [5], [1], 6], and [2]. All of these rely heavily on [11]. As the necessary facts about Radin forcing are distributed throughout the literature, our bibliographical citations will reflect this. 
Our witnessing model $N$ for Theorem 1(1) is the specific version of the model $N_{A}$ of 3] described at the end of that paper, except that $\lambda$ is now the least singular strong limit cardinal of cofinality $\omega$ above $\kappa$ instead of the least measurable cardinal above $\kappa$. (See also [2].) We explicitly give the construction below. Let $\lambda=\beth_{\omega}(\kappa)$. Let $j: V \rightarrow M$ be an elementary embedding witnessing the $2^{[\lambda]<\kappa}$ supercompactness of $\kappa$. Our first step is to define a Radin sequence of measures $\mu_{<\kappa^{+}}=\left\langle\mu_{\alpha} \mid \alpha<\kappa^{+}\right\rangle$appropriate for supercompact Radin forcing over $P_{\kappa}(\lambda)$. Specifically, if $\alpha=0, \mu_{\alpha}$ is given by $X \in \mu_{\alpha}$ iff $\langle j(\beta) \mid \beta<\lambda\rangle \in j(X)$, and if $\alpha>0, \alpha<\kappa^{+}, \mu_{\alpha}$ is given by $X \in \mu_{\alpha}$ iff $\left\langle\mu_{\beta} \mid \beta<\alpha\right\rangle={ }_{\text {df }} \mu_{<\alpha} \in j(X)$. Since $M^{2^{[\lambda]}<\kappa} \subseteq M, \mu_{<\kappa^{+}}$is well-defined.

Next, we let $\mathbb{R}_{<\kappa^{+}}$be supercompact Radin forcing over $P_{\kappa}(\lambda)$ defined using $\mu_{<\kappa^{+}}$. The particulars of the definition are virtually identical to the ones found in [5], [3], [1], [6], and [2], but for clarity, we repeat them here. $\mathbb{R}_{<\kappa^{+}}$is composed of all finite sequences of the form $\left\langle\left\langle p_{0}, u_{0}, C_{0},\right\rangle, \ldots,\left\langle p_{n}, u_{n}, C_{n}\right\rangle\right.$, $\left.\left\langle\mu_{<\kappa^{+}}, C\right\rangle\right\rangle$ such that the following conditions hold.

(1) For $0 \leq i<j \leq n, p_{i} \subsetneq p_{j}$, where for $p, q \in P_{\kappa}(\lambda), \quad p \subsetneq q$ means $p \subseteq q$ and $\operatorname{otp}(p)<q \cap \kappa$.

(2) For $0 \leq i \leq n, p_{i} \cap \kappa$ is a measurable cardinal.

(3) $\operatorname{otp}\left(p_{i}\right)$ is the least strong limit cardinal greater than $p_{i} \cap \kappa$ (which of course is $\left.\beth_{\omega}\left(p_{i} \cap \kappa\right)\right)$. In analogy to the notation of [1], [5], 3], [1], [6], and [2], we write $\operatorname{otp}\left(p_{i}\right)=\left(p_{i} \cap \kappa\right)^{*}$.

(4) For $0 \leq i \leq n, u_{i}$ is a Radin sequence of measures appropriate for supercompact Radin forcing over $P_{p_{i} \cap \kappa}\left(\operatorname{otp}\left(p_{i}\right)\right)$ with $\left(u_{i}\right)_{0}$, the 0th coordinate of $u_{i}$, a supercompact measure over $P_{p_{i} \cap \kappa}\left(\operatorname{otp}\left(p_{i}\right)\right)$.

(5) $C_{i}$ is a sequence of measure 1 sets for $u_{i}$.

(6) $C$ is a sequence of measure 1 sets for $\mu_{<\kappa^{+}}$.

(7) For each $p \in(C)_{0}$, where $(C)_{0}$ is the coordinate of $C$ such that $(C)_{0} \in \mu_{0}, \bigcup_{i \in\{0, \ldots, n\}} p_{i}=p_{n} \subsetneq p$.

(8) For each $p \in(C)_{0}$, otp $(p)=(p \cap \kappa)^{*}$ and $p \cap \kappa$ is a measurable cardinal.

Conditions (5) and (6) are both standard to any definition of Radin forcing. Conditions (1), (2), (4), and (7) are all standard to any definition of supercompact Radin forcing. Conditions (3) and (8) are used because of our ultimate aim of constructing a model in which the successor of every limit cardinal is singular of cofinality $\omega$. That they may be included and have the Radin forcing attain its desired goals follows by the fact that $V \vDash$ " $\kappa$ is supercompact and $\lambda$ is the least strong limit cardinal greater than $\kappa$ ". Thus, by closure, $M \vDash " \kappa$ is measurable and $\lambda$ is the least strong limit cardinal greater than $\kappa$ ". This means that by reflection, $\left\{p \in P_{\kappa}(\lambda) \mid p \cap \kappa\right.$ 
is a measurable cardinal and $\operatorname{otp}(p)$ is the least strong limit cardinal greater than $p \cap \kappa\} \in \mu_{0}$. This will ensure that the Radin sequence of cardinals eventually produced can be used in our final symmetric inner model $N$.

For completeness of exposition, we recall now the definition of the ordering on $\mathbb{R}_{<\kappa^{+}}$. If $\pi_{0}=\left\langle\left\langle p_{0}, u_{0}, C_{0}\right\rangle, \ldots,\left\langle p_{n}, u_{n}, C_{n}\right\rangle,\left\langle\mu_{<\kappa^{+}}, C\right\rangle\right\rangle$ and $\pi_{1}=$ $\left\langle\left\langle q_{0}, v_{0}, D_{0}\right\rangle, \ldots,\left\langle q_{m}, v_{m}, D_{m}\right\rangle,\left\langle\mu_{<\kappa^{+}}, D\right\rangle\right\rangle$, then $\pi_{1}$ extends $\pi_{0}$ iff the following conditions hold.

(1) For each $\left\langle p_{j}, u_{j}, C_{j}\right\rangle$ which appears in $\pi_{0}$, there is a $\left\langle q_{i}, v_{i}, D_{i}\right\rangle$ which appears in $\pi_{1}$ such that $\left\langle q_{i}, v_{i}\right\rangle=\left\langle p_{j}, u_{j}\right\rangle$ and $D_{i} \subseteq C_{j}$, i.e., for each coordinate $\left(D_{i}\right)_{\alpha}$ and $\left(C_{j}\right)_{\alpha},\left(D_{i}\right)_{\alpha} \subseteq\left(C_{j}\right)_{\alpha}$.

(2) $D \subseteq C$.

(3) $n \leq m$.

(4) If $\left\langle q_{i}, v_{i}, D_{i}\right\rangle$ does not appear in $\pi_{0}$, let $\left\langle p_{j}, u_{j}, C_{j}\right\rangle$ (or $\left\langle\mu_{<\kappa^{+}}, C\right\rangle$ ) be the first element of $\pi_{0}$ such that $p_{j} \cap \kappa>q_{i} \cap \kappa$. Then

(a) $q_{i}$ is order isomorphic to some $q \in\left(C_{j}\right)_{0}$.

(b) There exists an $\alpha<\alpha_{0}$, where $\alpha_{0}$ is the length of $u_{j}$, such that $v_{i}$ is isomorphic "in a natural way" to an ultrafilter sequence $v \in\left(C_{j}\right)_{\alpha}$.

(c) For $\beta_{0}$ the length of $v_{i}$, there is a function $f: \beta_{0} \rightarrow \alpha_{0}$ such that for $\beta<\beta_{0},\left(D_{i}\right)_{\beta}$ is a set of ultrafilter sequences such that for some subset $\left(D_{i}\right)_{\beta}^{\prime}$ of $\left(C_{j}\right)_{f(\beta)}$, each ultrafilter sequence in $\left(D_{i}\right)_{\beta}$ is isomorphic "in a natural way" to an ultrafilter sequence in $\left(D_{i}\right)_{\beta}^{\prime}$.

For further information on the definition of the ordering on $\mathbb{R}_{<\kappa^{+}}$(including the meaning of "in a natural way") and more facts about Radin forcing in general, readers are referred to [5], [3], [1], [6], [2], [8], [9], [1], [10], and [12].

We are now ready to define the partial ordering $\mathbb{P}$ used in the proof of Theorem 1(1). It is given by the finite support product ordered componentwise

$$
\prod_{\{\langle\alpha, \beta\rangle \mid \omega \leq \alpha<\beta<\kappa \text { are regular cardinals }\}} \operatorname{Coll}(\alpha,<\beta) \times \mathbb{R}_{<\kappa^{+}},
$$

where $\operatorname{Coll}(\alpha,<\beta)$ is the Lévy collapse of all cardinals of size less than $\beta$ to $\alpha$.

Let $G$ be $V$-generic over $\mathbb{P}$, and let $G_{0}$ be the projection of $G$ onto $\mathbb{R}_{<\kappa^{+}}$. For any condition $\pi \in \mathbb{R}_{<\kappa^{+}}$, call $\left\langle p_{0}, \ldots, p_{n}\right\rangle$ the p-part of $\pi$. Let $R=\{p \mid$ $\exists \pi \in G_{0}[p \in$ p-part $\left.(\pi)]\right\}$, and let $R_{\ell}=\{p \mid p \in R$ and $p$ is a limit point of $R\}$. Define three sets $E_{0}, E_{1}$, and $E_{2}$ by $E_{0}=\left\{\alpha \mid\right.$ For some $\pi \in G_{0}$ and some $p \in \operatorname{p-part}(\pi), p \cap \kappa=\alpha\}, E_{1}=\{\alpha<\kappa \mid \alpha$ is a limit point of $\left.E_{0}\right\}$, and $E_{2}=E_{1} \cup\{\omega\} \cup\left\{\beta \mid \exists \alpha\left[\alpha\right.\right.$ is a limit point of $E_{1}$ and $\left.\left.\beta=\alpha^{*}\right]\right\}$. Let $\left\langle\alpha_{\nu} \mid \nu<\kappa\right\rangle$ be the continuous, increasing enumeration of $E_{2}$, and let 
$\nu=\nu^{\prime}+n$ for some $n \in \omega$. For $\beta$ where $\beta \in\left[\alpha_{\nu}, \alpha_{\nu+1}\right)$ in the first case and $\beta=\alpha_{\nu+1}$ in the second and third cases, define sets $C_{i}\left(\alpha_{\nu}, \beta\right)$ for $i=1,2,3$ according to specific conditions on $\nu, \nu^{\prime}$, and $n$ in the following manner:

(1) $\nu=\nu^{\prime} \neq 0$ and $n=0$, i.e., $\nu$ is a limit ordinal. Let $p\left(\alpha_{\nu}\right)$ be the element $p$ of $R$ such that $p \cap \kappa=\alpha_{\nu}$, and let $h_{p\left(\alpha_{\nu}\right)}: p\left(\alpha_{\nu}\right) \rightarrow$ $\operatorname{otp}\left(p\left(\alpha_{\nu}\right)\right)$ be the order isomorphism between $p\left(\alpha_{\nu}\right)$ and $\operatorname{otp}\left(p\left(\alpha_{\nu}\right)\right)$. Then $C_{1}\left(\alpha_{\nu}, \beta\right)=\left\{h_{p\left(\alpha_{\nu}\right)}{ }^{\prime \prime} p \cap \beta \mid p \in R_{\ell}, p \subseteq p\left(\alpha_{\nu}\right)\right.$, and $h_{p\left(\alpha_{\nu}\right)}^{-1}(\beta)$ $\in p\}$.

(2) $\left(\nu=\nu^{\prime}+n, \nu^{\prime}>0\right.$, and $\left.2 \leq n<\omega\right)$ or $\left(\nu^{\prime}=0\right.$ and $\left.n \in \omega\right)$, i.e., $\nu$ is neither a limit ordinal nor the successor of a limit ordinal. Let $H\left(\alpha_{\nu}, \alpha_{\nu+1}\right)$ be the projection of $G$ onto $\operatorname{Coll}\left(\alpha_{\nu},<\alpha_{\nu+1}\right)$. Then $C_{2}\left(\alpha_{\nu}, \alpha_{\nu+1}\right)=H\left(\alpha_{\nu}, \alpha_{\nu+1}\right)$.

(3) $\nu=\nu^{\prime}+1$ for $\nu^{\prime}>0$, i.e., $\nu$ is the successor of a limit ordinal. Let $H\left(\alpha_{\nu}^{+}, \alpha_{\nu+1}\right)$ be the projection of $G$ onto $\operatorname{Coll}\left(\alpha_{\nu}^{+},<\alpha_{\nu+1}\right)$. Then $C_{3}\left(\alpha_{\nu}^{+}, \alpha_{\nu+1}\right)=H\left(\alpha_{\nu}^{+}, \alpha_{\nu+1}\right)$.

$C_{1}\left(\alpha_{\nu}, \beta\right)$ is used to collapse $\beta$ to $\alpha_{\nu}$ when $\nu$ is a limit ordinal, and is also used to generate the closed, cofinal sequence $\left\langle\alpha_{\gamma} \mid \gamma<\nu\right\rangle . C_{2}\left(\alpha_{\nu}, \alpha_{\nu+1}\right)$ is used to collapse $\alpha_{\nu+1}$ to be the successor of $\alpha_{\nu}$ when $\nu$ is neither a limit ordinal nor the successor of a limit ordinal, and $C_{3}\left(\alpha_{\nu}^{+}, \alpha_{\nu+1}\right)$ is used to collapse $\alpha_{\nu+1}$ to be the successor of $\alpha_{\nu}^{+}$when $\nu$ is the successor of a limit ordinal. Intuitively, the symmetric inner model $N \subseteq V[G]$ witnessing the conclusions of Theorem 1 1) is $V_{\kappa}$ of the least model of ZF extending $V$ which contains $C_{1}\left(\alpha_{\nu}, \beta\right)$ if $\nu$ is a limit ordinal and $\beta \in\left[\alpha_{\nu}, \alpha_{\nu+1}\right), C_{2}\left(\alpha_{\nu}, \alpha_{\nu+1}\right)$ if $\nu$ is neither a limit ordinal nor the successor of a limit ordinal, and $C_{3}\left(\alpha_{\nu}^{+}, \alpha_{\nu+1}\right)$ if $\nu$ is the successor of a limit ordinal.

To define $N$ more precisely, it is necessary to define canonical names $\underline{\alpha_{\nu}}$ for the $\alpha_{\nu}$ 's and canonical names $C_{i}(\nu, \beta)$ for $i=1$ and $C_{i}(\nu, \nu+1)$ for $i=$ 2,3 . Recall that it is possible to decide $p\left(\alpha_{\nu}\right)$ (and hence otp $\left(p\left(\alpha_{\nu}\right)\right)$ ) by writing $\omega \cdot \nu=\omega^{\sigma_{0}} \cdot n_{0}+\omega^{\sigma_{1}} \cdot n_{1}+\cdots+\omega^{\sigma_{m}} \cdot n_{m}$ (where $\sigma_{0}>\sigma_{1}>\cdots>\sigma_{m}$ are ordinals, $n_{0}, \ldots, n_{m}>0$ are integers, and,$+ \cdot$, and exponentiation are the ordinal arithmetical operations), letting $\pi=\left\langle\left\langle p_{i j_{i}}, u_{i j_{i}}, C_{i j_{i}}\right\rangle_{i \leq m, 1 \leq j_{i} \leq n_{i}},\left\langle\mu_{<\kappa^{+}}, C\right\rangle\right\rangle$ be such that $\min \left(p_{i 1} \cap \kappa, \omega^{\text {length }\left(u_{i 1}\right)}\right)=\sigma_{i}$ and length $\left(u_{i j_{i}}\right)=\min \left(p_{i 1} \cap \kappa\right.$, length $\left.\left(u_{i 1}\right)\right)$ for $1 \leq j_{i} \leq n_{i}$, and letting $p\left(\alpha_{\nu}\right)$ be $p_{m n_{m}}$. Further, $D_{\nu}=\{r \in$ $\mathbb{P}|r| \mathbb{R}_{<\kappa^{+}}$extends a condition $\pi$ of the above form $\}$ is a dense open subset of $\mathbb{P} . \alpha_{\nu}$ is the name of the $\alpha_{\nu}$ determined by any element of $D_{\nu} \cap G$; in the notation of [11, [5], 3], 11, [6], and [2], $\alpha_{\nu}=\left\{\left\langle r, \check{\alpha}_{\nu}(r)\right\rangle \mid r \in D_{\nu}\right\}$, where $\alpha_{\nu}(r)$ is the $\alpha_{\nu}$ determined by the condition $r$.

The canonical names $C_{i}(\nu, \beta)$ for $i=1$ and $C_{i}(\nu, \nu+1)$ for $i=2,3$ are defined in a manner so as to be invariant under the appropriate group of automorphisms. Specifically, there are three cases to consider. We again write $\nu=\nu^{\prime}+n$, where $n \in \omega$ and $\nu^{\prime}$ is either a limit ordinal or 0 , and let $\beta$ 
be as before. We also assume without loss of generality that as in [11, [5], [3], 1], 6], and [2], $\alpha_{\nu+1}$ is determined by $D_{\nu}$. Further, we adopt throughout each of the three cases the notation of [11], [5], [3], 1], [6], and [2].

(1) $\nu^{\prime}=\nu \neq 0$ and $n=0$, i.e., $\nu$ is a limit ordinal. Then $C_{1}(\nu, \beta)=$ $\left\{\left\langle r,(\check{r}\rceil \mathbb{R}_{<\kappa^{+}}\right)\left\lceil\left(\alpha_{\nu}(r), \beta\right)\right\rangle \mid r \in D_{\nu}\right\}$, where for $r \in \mathbb{P}, \pi \overline{=r \uparrow \mathbb{R}_{<\kappa^{+}}}$, $\pi \uparrow\left(\alpha_{\nu}(r), \beta\right)=\left\{h_{p\left(\alpha_{\nu}\right)(r)}{ }^{\prime \prime} p \cap \beta \mid p \in \operatorname{p}-\operatorname{part}(\pi), p \subseteq p\left(\alpha_{\nu}\right)(r), p \in\right.$ $R_{\ell}\left\lceil\pi\right.$, and $\left.h_{p\left(\alpha_{\nu}\right)(r)}^{-1}(\beta) \in p\right\}$.

(2) $\left(\nu=\nu^{\prime}+n, \nu^{\prime}>0\right.$, and $\left.2 \leq n<\omega\right)$ or $\left(\nu^{\prime}=0\right.$ and $\left.n \in \omega\right)$, i.e., $\nu$ is neither a limit ordinal nor the successor of a limit ordinal. Then $\underline{C_{2}(\nu, \nu+1)}=\left\{\left\langle r,\left(\check{r}\left\lceil\operatorname{Coll}\left(\alpha_{\nu}(r),<\alpha_{\nu+1}(r)\right)\right)\right\rangle\right| r \in D_{\nu}\right\}$.

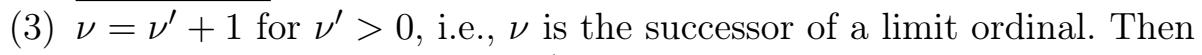
$C_{3}(\nu, \nu+1)=\left\{\left\langle r,\left(\check{r} \mid \operatorname{Coll}\left(\alpha_{\nu}^{+}(r),<\alpha_{\nu+1}(r)\right)\right)\right\rangle \mid r \in D_{\nu}\right\}$.

As in [11], [5], [3], 1], 6], and [2], since for any $r, r^{\prime} \in D_{\nu} \cap G, p\left(\alpha_{\nu}\right)(r)=$ $p\left(\alpha_{\nu}\right)\left(r^{\prime}\right)$, each of the definitions just given is unambiguous.

Let $\mathcal{G}$ be the group of automorphisms of [11]. Set $C(G)=\left\{\psi\left(C_{1}(\nu, \beta)\right) \mid\right.$ $\psi \in \mathcal{G}, 0 \leq \nu<\kappa$, and $\beta \in[\nu, \kappa)$ is a cardinal $\} \cup \bigcup \overline{i=2,3}\left\{\psi\left(\overline{C_{i}(\overline{\nu, \nu+1)})}\right)\right.$ $\psi \in \mathcal{G}$ and $0 \leq \nu<\kappa\}$. Then $C(G)=\left\{i_{G}\left(\psi\left(C_{1}(\nu, \beta)\right)\right) \mid \psi \in \overline{\mathcal{G}, 0 \leq \nu<\kappa}\right.$, and $\beta \in[\nu, \kappa)$ is a cardinal $\} \cup \bigcup_{i=2,3}\left\{i_{G}\left(\psi\left(\overline{C_{i}(\nu, \nu+1}\right)\right)\right) \mid \psi \in \mathcal{G}$ and $0 \leq$ $\nu<\kappa\}=i_{G}(C(G)) . N$ is then the set of all sets of rank less than $\kappa$ of the model consisting of all sets which are hereditarily $V$ definable from $C(G)$, i.e., $N=V_{\kappa}^{\mathrm{HVD}(C(G))}$.

Let $\left\langle\delta_{\nu} \mid \nu<\kappa\right\rangle$ be the continuous, increasing enumeration of $\left\{\alpha_{\nu} \mid \nu<\kappa\right\}$ $\cup\left\{\left(\alpha_{\nu}^{+}\right)^{V} \mid \nu<\kappa\right.$ is the successor of a limit ordinal $\}$. The arguments of [1] and [3, Lemma 1] allow us to conclude that $N \vDash$ "ZF $+\neg \mathrm{AC}_{\omega}+$ For every limit ordinal $\nu, \delta_{\nu}=\alpha_{\nu}=\aleph_{\nu}$ is singular + If $\nu=\nu^{\prime}+1$ and $\nu^{\prime}$ is not a limit ordinal, then $\delta_{\nu}$ is a regular cardinal $+\forall \nu\left[\delta_{\nu} \leq \aleph_{\nu}\right]$ ". In addition, we know that for any ordinal $\gamma$ and any set $x \subseteq \gamma, x \in N, x=\{\alpha<\gamma \mid$ $\left.V[G] \vDash \phi\left(\alpha, i_{G}\left(\psi_{1}\left(C_{i_{1}}\left(\nu_{1}, \beta_{1}\right)\right)\right), \ldots, i_{G}\left(\psi_{n}\left(C_{i_{n}}\left(\nu_{n}, \beta_{n}\right)\right)\right), C(G)\right)\right\}$, where $i_{j}$ is an integer, $1 \leq j \leq n, 1 \leq i_{j} \leq 3$, each $\psi_{i} \in \mathcal{G}$, each $\beta_{i}$ is an appropriate ordinal for $\nu_{i}$, and $\phi\left(x_{0}, \ldots, x_{n+1}\right)$ is a formula which may also contain some parameters from $V$ which we shall suppress.

Let

$$
\overline{\mathbb{P}}=\prod_{i_{j}=2, j \leq n} \operatorname{Coll}\left(\alpha_{\nu_{j}},<\alpha_{\nu_{j}+1}\right) \times \prod_{i_{j}=3, j \leq n} \operatorname{Coll}\left(\alpha_{\nu_{j}}^{+},<\alpha_{\nu_{j}+1}\right) \times \mathbb{R}_{<\kappa^{+}} .
$$

For $\pi \in \mathbb{R}_{<\kappa^{+}}$, let $\pi\left\lceil\gamma=\{\langle q, u, C\rangle \in \pi \mid q \cap \kappa \leq \gamma\}\right.$, and let $\mathbb{R}_{\gamma}=$ $\left\{\pi|\gamma| \pi \in \mathbb{R}_{<\kappa^{+}}\right\}$. For $p \in \mathbb{\mathbb { P }}, p=\left\langle p_{1}, \ldots, p_{m}, \pi\right\rangle, m \leq n, \pi \in \mathbb{R}_{<\kappa^{+}}$, let $p\left\lceil\gamma=\left\langle q_{1}, \ldots, q_{m}, \pi\lceil\gamma\rangle\right.\right.$, where $q_{j}=p_{j}$ if $\alpha_{\nu_{j}} \leq \gamma$ and $q_{j}=\emptyset$ otherwise. In other words, $p\lceil\gamma$ is the part of $p$ below or at $\gamma$. Without loss of generality, we ignore the empty coordinates and let $\overline{\mathbb{P}}\lceil\gamma=\{p\lceil\gamma \mid p \in \overline{\mathbb{P}}\}$. Let $G\lceil\gamma$ be the 
projection of $G$ onto $\overline{\mathbb{P}}\lceil\gamma$. An analogous fact to [11, Theorem 3.2.11] holds, using the same proof as in [11, namely $x \in V[G\lceil\gamma]$. In addition, the elements of $\overline{\mathbb{P}} \gamma \gamma$ can be partitioned into equivalence classes (the "almost similar" equivalence classes of [11]) with respect to $C_{i_{1}}\left(\nu_{1}, \beta_{1}\right), \ldots, C_{i_{n}}\left(\nu_{n}, \beta_{n}\right)$ via an equivalence relation to be called $\sim$ such that if $\varphi$ is any formula mentioning only (terms for ground model sets and) $C_{i_{1}}\left(\nu_{1}, \beta_{1}\right), \ldots, C_{i_{n}}\left(\nu_{n}, \beta_{n}\right)$, and $C(G), p \| \varphi$ (i.e., $p$ decides $\varphi$ ), and $q \sim p$, then $q \| \varphi$ in the same way that $p$ does. It thus follows as an immediate corollary of the work of [11 that if we define $G^{\left\langle\left\langle\nu_{1}, \beta_{1}\right\rangle, \ldots,\left\langle\nu_{n}, \beta_{n}\right\rangle\right\rangle}=\left\{[p]_{\sim} \mid p \in G\lceil\gamma\}\right.$, then $x \in V\left[G^{\left\langle\left\langle\nu_{1}, \beta_{1}\right\rangle, \ldots,\left\langle\nu_{n}, \beta_{n}\right\rangle\right\rangle}\right]$ and $V\left[G\left\langle\left\langle\nu_{1}, \beta_{1}\right\rangle, \ldots,\left\langle\nu_{n}, \beta_{n}\right\rangle\right\rangle\right] \subseteq N$. It further follows by the work of [11] that if $\nu<\kappa$ is a limit ordinal and $\gamma=\alpha_{\nu+1}$, then since $\delta_{\nu+1}=\alpha_{\nu+1}=\alpha_{\nu}^{*}$ and $\alpha_{\nu}^{*}$ is a strong limit cardinal, there are fewer than $\alpha_{\nu+1}$ many such equivalence classes. In other words, $G^{\left\langle\left\langle\nu_{1}, \beta_{1}\right\rangle, \ldots,\left\langle\nu_{n}, \beta_{n}\right\rangle\right\rangle}$ is $V$-generic over a partial ordering forcing equivalent to a partial ordering $\mathbb{Q}^{\left\langle\left\langle\nu_{1}, \beta_{1}\right\rangle, \ldots,\left\langle\nu_{n}, \beta_{n}\right\rangle\right\rangle}$ such that $\mid \mathbb{Q}^{\left\langle\left\langle\nu_{1}, \beta_{1}\right\rangle, \ldots,\left\langle\nu_{n}, \beta_{n}\right\rangle\right\rangle \mid}\left\langle\alpha_{\nu+1}\right.$. It is this last fact, in tandem with the way in which $N$ is defined, which allows us to show that $N$ is our desired model. In particular, the following two lemmas complete the proof of Theorem 1(1).

Lemma 1.1. If $\nu<\kappa$ is a limit ordinal, then $N \vDash " \alpha_{\nu+1}$ is a singular cardinal having cofinality $\omega "$.

Proof. Since $\nu<\kappa$ is a limit ordinal, as was mentioned above, $\delta_{\nu+1}=$ $\alpha_{\nu+1}=\alpha_{\nu}^{*}$. By the definition of $\alpha_{\nu}^{*}, V \vDash " \alpha_{\nu}^{*}$ is a strong limit cardinal having cofinality $\omega "$ ", so since $V \subseteq N, N \vDash " \operatorname{cof}\left(\alpha_{\nu+1}\right)=\omega "$ ". Then, to see that $N \vDash " \alpha_{\nu+1}$ is a cardinal", suppose that $\rho<\alpha_{\nu+1}$ and $N \vDash " f: \rho \rightarrow$ $\alpha_{\nu+1}$ is a function". Since $f$ may be coded by a subset of $\alpha_{\nu+1}$, by the preceding paragraph, $f \in V\left[G\left(\left\langle\nu_{1}, \beta_{1}\right\rangle, \ldots,\left\langle\nu_{n}, \beta_{n}\right\rangle\right\rangle\right] \subseteq N$ for the appropriate generic object $G\left\langle\left\langle\nu_{1}, \beta_{1}\right\rangle, \ldots,\left\langle\nu_{n}, \beta_{n}\right\rangle\right\rangle$. Because $G\left\langle\left\langle\nu_{1}, \beta_{1}\right\rangle, \ldots,\left\langle\nu_{n}, \beta_{n}\right\rangle\right\rangle$ is $V$-generic over a partial ordering forcing equivalent to a partial ordering having cardinality less than $\alpha_{\nu+1}, f$ cannot witness that $\alpha_{\nu+1}$ is no longer a cardinal. This completes the proof of Lemma 1.1 .

Lemma 1.2. $N \vDash$ "All successor cardinals except successors of uncountable limit cardinals are regular + Every uncountable limit cardinal is singular + The successor of every uncountable limit cardinal is singular of cofinality $\omega "$.

Proof. By our earlier remarks, $N \vDash " \delta_{\nu}$ is a cardinal, except possibly if $\nu=\nu^{\prime}+1$ and $\nu^{\prime}$ is a limit ordinal". By Lemma 1.1. for such a $\nu, N \vDash$ " $\delta_{\nu}=\alpha_{\nu}$ is a singular cardinal of cofinality $\omega "$. Since as we have already observed, $N \vDash$ " $\forall \nu\left[\delta_{\nu} \leq \aleph_{\nu}\right]$ ", an easy induction in conjunction with the fact that $N \vDash$ "For every limit ordinal $\nu, \delta_{\nu}=\alpha_{\nu}=\aleph_{\nu}$ is singular + If $\nu=\nu^{\prime}+1$ and $\nu^{\prime}$ is not a limit ordinal, then $\delta_{\nu}$ is a regular cardinal" now shows that $N \vDash " \forall \nu\left[\delta_{\nu}=\aleph_{\nu}\right]$ ". In particular, we may also infer that 
$N \vDash$ "All successor cardinals except successors of uncountable limit cardinals are regular + Every uncountable limit cardinal is singular + The successor of every uncountable limit cardinal is singular of cofinality $\omega "$. This completes the proof of Lemma 1.2 .

Lemmas 1.1 and 1.2 complete the proof of Theorem 1 1 (1).

To prove Theorem 1(2) (and thereby complete the proof of Theorem 1), we assume $\mathrm{ZF}$ and that all uncountable limit cardinals are singular and the successor of every uncountable limit cardinal is singular. Let $\Phi(\rho)$ be the statement " $\rho$ is a singular cardinal". Since ZFC $\vdash$ "All successor cardinals are regular", if $\Phi(\rho)$ holds, there is no inner model of ZFC in which $\rho$ is a successor cardinal. Further, by assumption, there is a proper class of cardinals $\delta$ in which $\delta$ is singular and $\Phi\left(\delta^{+}\right)$is true. Therefore, by [6, Theorem 5] (see also the work of [7, Section 3.1], from which [6, Theorem 5] is derived), for every $n<\omega$ and every set of ordinals $x, M_{n}^{\sharp}(x)$ exists. This completes the proof of both Theorem 1 $(2)$ and Theorem 1.

To sketch the proof of Theorem 2, suppose $V \vDash$ "ZFC $+\kappa$ is $2^{\left[\beth_{\omega_{1}}(\kappa)\right]^{<\kappa}}$ supercompact". Let $\lambda=\beth_{\omega_{1}}(\kappa)$, and let $\mu_{<\kappa^{+}}$and $\mathbb{R}_{<\kappa^{+}}$be defined exactly as in the proof of Theorem $1(1)$ using this value of $\lambda$. Define $\mathbb{P}$ as the finite support product ordered componentwise

$$
\prod_{\left\{\langle\alpha, \beta\rangle \mid \omega_{1} \leq \alpha<\beta<\kappa \text { are regular cardinals }\right\}} \operatorname{Coll}(\alpha,<\beta) \times \mathbb{R}_{<\kappa^{+}},
$$

i.e., $\mathbb{P}$ is defined as in the proof of Theorem $1(1)$, except that the smallest regular cardinal to which another regular cardinal may be collapsed is $\left(\omega_{1}\right)^{V}\left({ }^{1}\right)$. Since this definition of $\mathbb{P}$ ensures that $\left(\omega_{1}\right)^{V}$ is not collapsed (and so remains a regular cardinal), if we construct $N$ in the analogous manner to the construction given in the proof of Theorem 1(1), we may use the same arguments as given in the proof of Theorem 1 1(1) to prove Theorem 2(1). The proof of Theorem 2(2) is then exactly the same as the proof of Theorem 1(2). This completes the proof sketch of Theorem 2.

We note that in the proof of Theorem 2, there is nothing special about cofinality $\omega_{1}$. Other uncountable cofinalities, e.g., $\omega_{2}, \omega_{3}$, etc. are also possible, with the appropriate further modifications analogous to those given in the proof of Theorem 2. We leave it to interested readers to work out the details for themselves.

There is of course a vast disparity between the upper and lower bounds in consistency strength given in Theorems 1 and 2 . We conclude by asking if it is possible to prove equiconsistencies for both of these theorems, and

$\left({ }^{1}\right)$ This idea was also used in the proof of the main theorem of [2]. 
not just "sandwich theorems". Unfortunately, such results remain outside the reach of current set-theoretic technology.

Acknowledgments. The author's research was partially supported by PSC-CUNY grants.

\section{References}

[1] A. Apter, A cardinal pattern inspired by AD, Math. Logic Quart. 42 (1996), 211-218.

[2] -, How many normal measures can $\aleph_{\omega_{1}+1}$ carry?, ibid. 56 (2010), to appear.

[3] - On the class of measurable cardinals without the axiom of choice, Israel J. Math. 79 (1992), 367-379.

[4] - Some new upper bounds in consistency strength for certain choiceless large cardinal patterns, Arch. Math. Logic 31 (1992), 201-205.

[5] - Some results on consecutive large cardinals II: Applications of Radin forcing, Israel J. Math. 52 (1985), 273-292.

[6] A. Apter and P. Koepke, Making all cardinals almost Ramsey, Arch. Math. Logic 47 (2008), 769-783.

[7] D. Busche and R. Schindler, The strength of choiceless patterns of singular and weakly compact cardinals, Ann. Pure Appl. Logic 159 (2009), 198-248.

[8] J. Cummings and W. H. Woodin, Generalised Prikry forcings, circulated manuscript.

[9] M. Foreman and W. H. Woodin, The GCH can fail everywhere, Ann. of Math. 133 (1991), 1-36.

[10] M. Gitik, Prikry-type forcings, forthcoming article in the Handbook of Set Theory.

[11] -, Regular cardinals in models of ZF, Trans. Amer. Math. Soc. 290 (1985), 41-68.

[12] L. Radin, Adding closed cofinal sequences to large cardinals, Ann. Math. Logic 23 (1982), 263-283.

Arthur W. Apter

Department of Mathematics

Baruch College of CUNY

New York, NY 10010, U.S.A.

and

The CUNY Graduate Center, Mathematics

365 Fifth Avenue

New York, NY 10016, U.S.A.

E-mail: awapter@alum.mit.edu

http://faculty.baruch.cuny.edu/apter

Received October 21, 2009;

received in final form November 21, 2009 